\title{
Surface membrane staining of immunoglobulins in paraffin sections of non-Hodgkin's lymphomas using immunogold-silver staining technique
}

\author{
CS HOLGATE, P JACKSON, I LAUDER, PN COWEN, CC BIRD \\ From the Department of Pathology, University of Leeds, Leeds
}

SUMMARY The immunogold-silver staining (IGSS) method is a new immunostaining technique with much enhanced sensitivity for demonstration of antigens in paraffin sections. A series of 10 non-Hodgkin's lymphomas of B cell type were stained for surface membrane immunoglobulins by the IGSS and peroxidase-antiperoxidase (PAP) methods using paraffin sections and polyclonal primary antisera. The resulting staining patterns were compared with those obtained using frozen sections of the same tissues, monoclonal antibodies and the immunoperoxidase technique. The IGSS method gave a clear demonstration of surface membrane immunoglobulins in neoplastic lymphocytes using paraffin sections and the pattern of staining achieved was comparable to that obtained by the immunoperoxidase technique employing frozen sections and monoclonal antibodies. PAP staining of paraffin sections consistently failed to demonstrate the presence of any surface membrane immunoglobulin. The IGSS method provides a new approach to the diagnosis of B cell lymphomas in which routinely fixed and processed tissues may be employed to demonstrate monoclonality.

A new method of immunostaining-immunogoldsilver staining (IGSS)--has recently been described ${ }^{1}$ which exhibits much enhanced sensitivity over standard peroxidase-antiperoxidase (PAP) techniques for demonstration of cytoplasmic immunoglobulins. We report here the results of further studies where the application of this new method for demonstrating surface membrane immunoglobulins in paraffin sections of non-Hodgkin's lymphomas has been investigated. The findings are compared with staining of the same tissues by conventional immunoperoxidase techniques using polyclonal and monoclonal antibodies. The results show that the IGSS method provides a simple and reliable technique for demonstrating surface membrane immunoglobulins in paraffin sections of nonHodgkin's lymphomas.

\section{Material and methods}

TISSUE

Lymph node biopsies from 10 cases diagnosed on routine haematoxylin and eosin stained sections

Accepted for publication 30 March 1983 according to the British National Lymphoma Investigation (BNLI) classification as non-Hodgkin's lymphoma and thought to be of B cell type were selected for study. Each specimen was received fresh (unfixed) within half an hour of removal. From each biopsy a piece of tissue was taken for immediate freezing and storage in liquid nitrogen. Another piece was fixed for $24 \mathrm{~h}$ in formol sublimate $(90 \mathrm{ml}$ saturated aqueous mercuric chloride plus $10 \mathrm{ml}$ of $40 \%$ aqueous formaldehyde) and processed routinely to paraffin wax.

\section{IMMUNOHISTOLOGICAL REAGENTS}

Polyclonal rabbit antisera to human IgA, IgG, IgM, IgD, $\kappa$ and $\lambda$ chains were obtained from Dakopatts (Mercia Brocades, Weybridge, Surrey). Mouse monoclonal antibodies to human IgA, IgG, IgM, IgD, $\kappa$ and $\lambda$ chains were obtained from Seward Laboratory (London). Swine antirabbit immunoglobulins, rabbit peroxidase-antiperoxidase complex and rabbit antimouse immunoglobulins conjugated to horseradish peroxidase were obtained from Dakopatts. Diaminobenzidine tetrahydrochloride and 3-amino-9-ethylcarbazole were obtained from Sigma London Chemical Co Ltd (Poole, Dorset). 
IGSS METHOD

Paraffin sections $(5 \mu \mathrm{m})$ were stained by this method as previously described.' Briefly, sections were treated with iodine and sodium thiosulphate, blocked with undiluted normal swine serum (NSS) and incubated with primary (polyclonal) antiserum raised in rabbits against the human immunoglobulin under study. These were diluted $1 / 1000$ in $5 \%$ NSS for demonstration of $\operatorname{IgA}, \operatorname{IgG}, \operatorname{IgM}, \kappa$ and $\lambda$ chains and 1/50 for IgD. After washing, a further block with NSS was applied and sections were then incubated overnight with the immunogold reagent. The immunogold reagent consisted of colloidal gold (particle size $\simeq 20 \mathrm{~nm}$ ) adsorbed with swine antirabbit immunoglobulins. In this way the immunogold reagent interacted specifically with the primary antiserum and gold particles were introduced to antigenic sites. After a further washing procedure the sections were immersed in the developing solution and the gold particles localised at antigenic sites were subsequently revealed under light microscopical control by a silver precipitation reaction. In this instance the immunogold reagent was used at a tenfold higher concentration than previously described ${ }^{\prime}$ and the sections were counterstained with $0.5 \%$ safranin in $0.125 \mathrm{~N}$ hydrochloric acid.

\section{PAP METHOD}

Paraffin sections $(5 \mu \mathrm{m})$ were stained by this method as previously described ${ }^{1}$ using 3amino-9-ethylcarbazole as the chromogenic substrate. Sections were counterstained with haematoxylin. The optimal concentrations of primary (polyclonal) antisera were the same as for the IGSS method.

\section{INDIRECT IMMUNOPEROXIDASE METHOD}

The indirect immunoperoxidase method applied to frozen sections is detailed below. The monoclonal antibodies were used diluted 1/1000 for demonstration of $\operatorname{IgG}, \kappa$ and $\lambda$ chains, $1 / 100$ for $\operatorname{IgA}, 1 / 5$ for IgM and undiluted for IgD.

\section{Indirect immunoperoxidase method for monoclonal} antibody staining

1 Cut $8 \mu \mathrm{m}$ frozen sections and freeze dry for $24 \mathrm{~h}$.

2 Fix in acetone for $15 \mathrm{~min}$.

3 Rehydrate in $0.2 \mathrm{M}$ sodium cacodylate in Trisbuffered saline $(0.05 \mathrm{M}$ Tris in isotonic saline, $\mathrm{pH} 7 \cdot 6$ ) for 1-2 $\mathrm{h}$.

4 Incubate with 5\% normal rabbit serum for $15 \mathrm{~min}$, remove excess and apply monoclonal antibody appropriately diluted in Tris-buffered saline for $1 \mathrm{~h}$.

5 Rinse each section separately in Tris-buffered saline and then wash for 10 min with two changes of buffer.

6 Treat sections with rabbit antimouse horseradish peroxidase conjugate diluted $1 / 25$ in $5 \%$ human $A B$ serum for $30 \mathrm{~min}$.

7 Rinse in Tris-buffered saline for $5 \mathrm{~min}$ and develop with diaminobenzidine $(150 \mathrm{mg}$ in $300 \mathrm{ml}$ Tris-buffered saline) for 5-10 min after addition of 10 drops of hydrogen peroxide $(30 \% \mathrm{wt} / \mathrm{vol})$ from a Pasteur pipette.

8 Wash in water $(2 \mathrm{~min})$ and incubate in $0.5 \%$ copper sulphate solution (in $0.9 \%$ saline) for $5 \mathrm{~min}$. 9 Wash and counterstain in $2 \%$ aqueous methyl green washed in chloroform for $30 \mathrm{~s}$.

10 Wash well, dehydrate, clear and mount in synthetic mounting medium.

\section{Results}

Staining of paraffin sections by the PAP method using polyclonal primary antisera on paraffin sections failed to reveal surface membrane immunoglobulins in neoplastic lymphocytes of any cases, although in most cases variable numbers of plasma cells were demonstrated. Since these were positive for both $\kappa$ and $\lambda$ light chains they were considered to be of polyclonal origin and not part of the neoplastic proliferation. By contrast, the IGSS method (using polyclonal antisera) applied to paraffin sections and the indirect immunoperoxidase method (using monoclonal antibodies) applied to frozen sections showed evidence of light chain restriction in every case studied indicating the monoclonal nature of these B cell proliferations. The type of staining achieved is illustrated in Figs. 1-4. When reacted for $\kappa$ chains the majority of neoplastic cells are surrounded by a thick deposit of stain which is considered localised to the surface membrane. By contrast, staining for $\lambda$ chains failed to reveal the presence of any surface membrane immunoglobulin. Staining for heavy chains showed the majority of cells were positive for IgA and the IGSS method revealed also the presence of IgM although this was not seen with immunoperoxidase stains.

The results of immunostaining the 10 lymphomas by the two techniques are summarised in the Table. It can be seen that the monoclonal nature of the $B$ cell proliferation was confirmed in each case by both methods and excellent agreement was obtained in the light and heavy chains demonstrated. In one instance (case 8) the IGSS method revealed the presence of an additional heavy chain.

\section{Discussion}

The superior sensitivity of the IGSS method over 

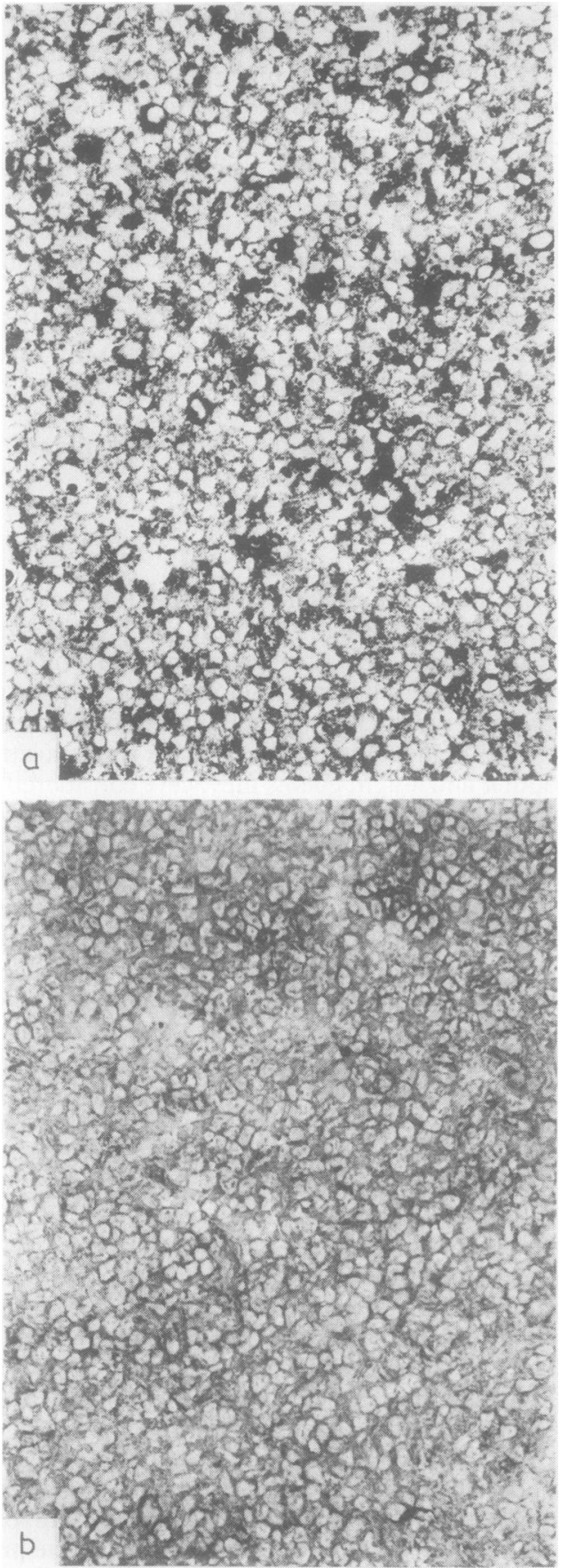

Fig. 1 Case No 8. Diffuse large cell lymphoma (immunoblastic type), stained for $\kappa$ chains by (a) IGSS method (paraffin section). Counterstain safranin $\times 200$. (b) Immunoperoxidase method (frozen section). Counterstain methyl green $\times 200$. Most cells show positive surface membrane staining by both methods.
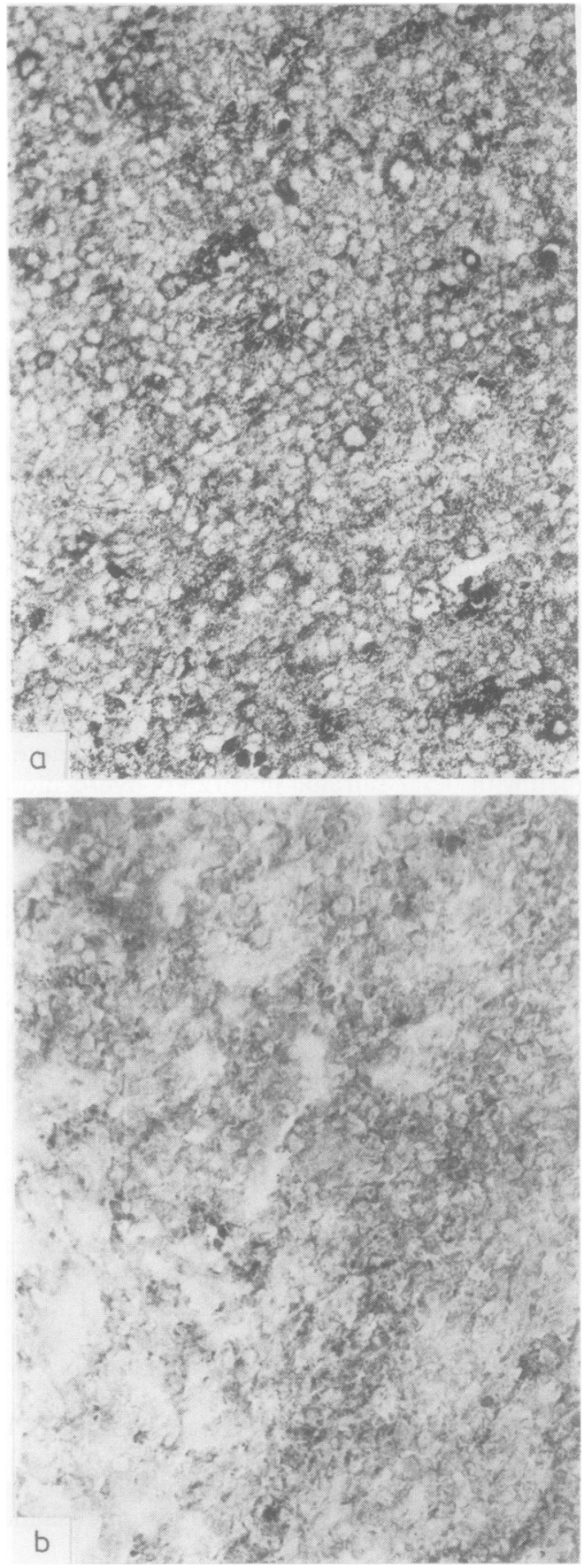

Fig. 2 Case No 8 stained for $\lambda$ chains by (a) IGSS method (paraffin section). Counterstain safranin $\times 200$.

(b) Immunoperoxidase method (frozen section).

Counterstain methyl green $\times 200$. Both methods show background staining only. 

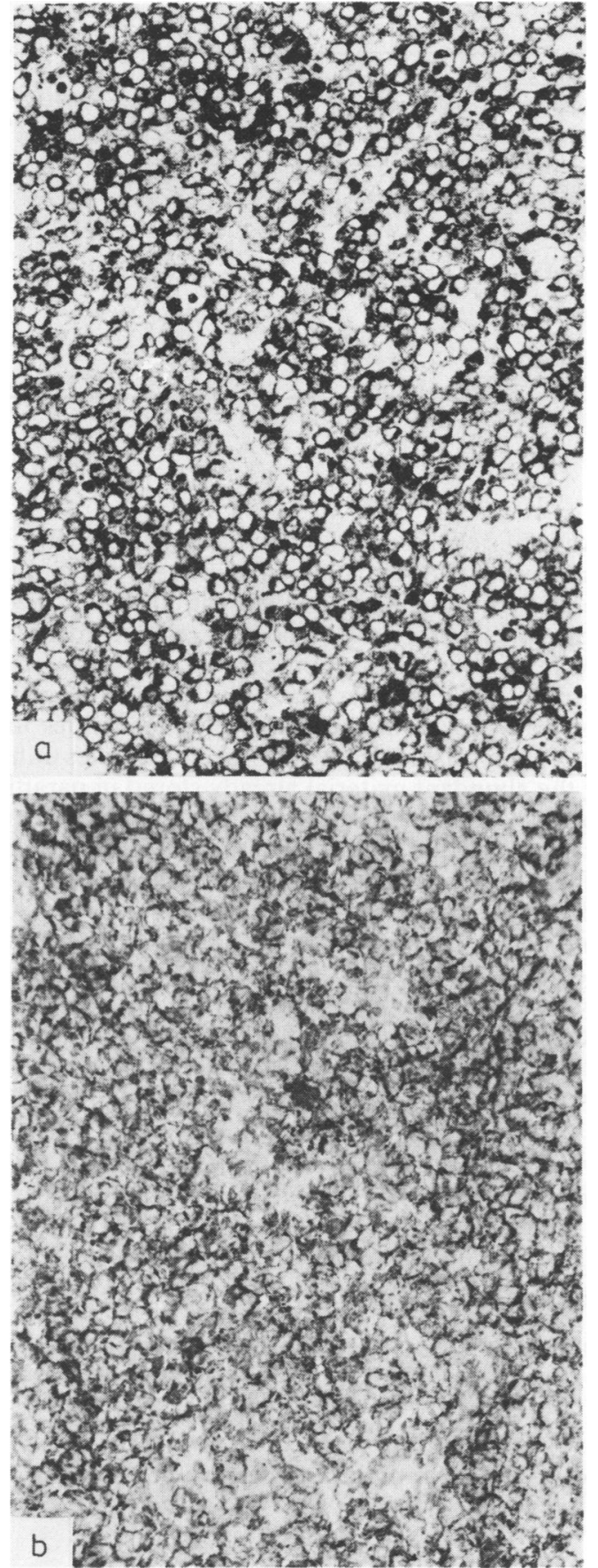

Fig. 3 Case No 8 stained for IgA by (a) IGSS method (paraffin section). Counterstain safranin $\times 200$. (b) Immunoperoxidase method (frozen section). Counterstain methyl green $\times 200$. Most cells show positive surface membrane staining by both methods.
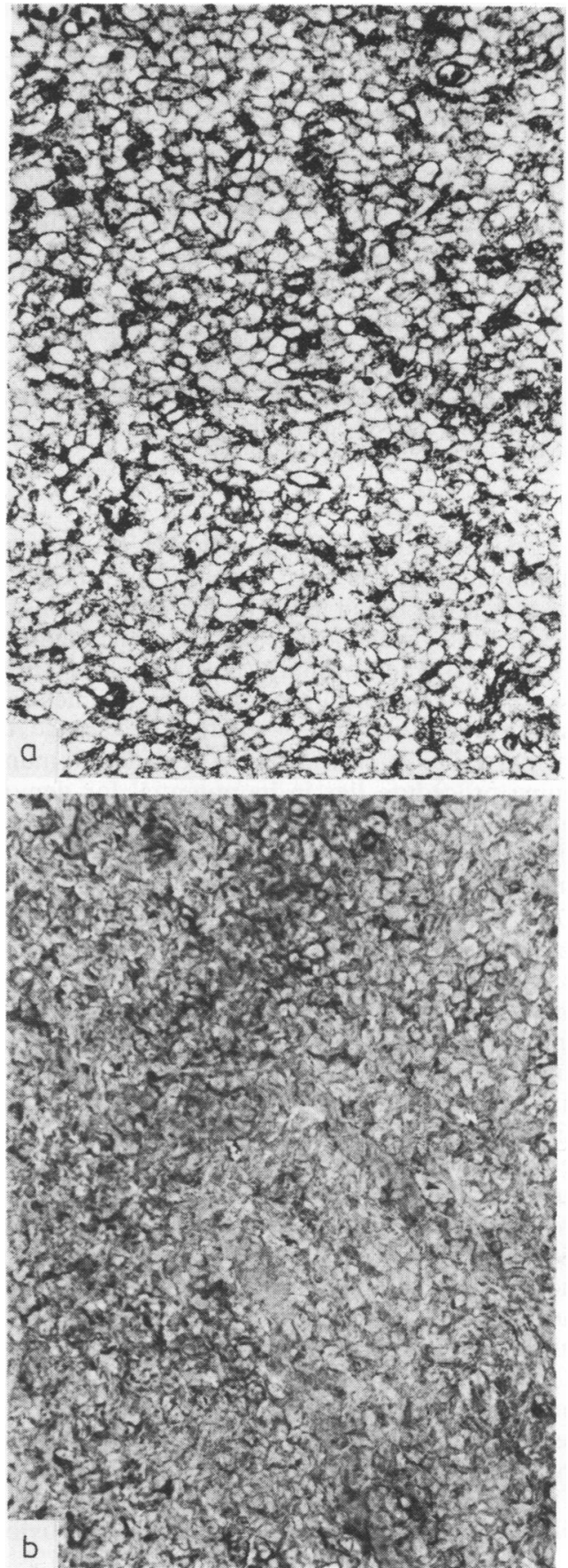

Fig. 4 Case No 8 stained for IgM by (a) IGSS method (paraffin section). Counterstain safranin $\times 200$. (b) Immunoperoxidase method (frozen section). Counterstain methyl green $\times 200$. Most cells show positive surface membrane staining by IGSS method but only background staining with immunoperoxidase method. 
Surface membrane immunoglobulin staining of non-Hodgkin's lymphomas in paraffin (IGSS) and frozen (immunoperoxidase) sections

\begin{tabular}{|c|c|c|c|c|c|c|}
\hline \multirow[t]{2}{*}{ Case No } & \multirow[t]{2}{*}{ Diagnosis } & \multirow[t]{2}{*}{ Sex } & \multirow[t]{2}{*}{ Age (yr) } & \multirow{2}{*}{$\begin{array}{l}\text { Lymph node } \\
\text { biopsied }\end{array}$} & \multicolumn{2}{|c|}{ Immunoglobulins demonstrated } \\
\hline & & & & & IGSS method* & $\begin{array}{l}\text { Immunoperoxidase } \\
\text { method } \dagger\end{array}$ \\
\hline $\begin{array}{r}1 \\
2 \\
3 \\
4 \\
5 \\
6 \\
7 \\
8 \\
9 \\
10\end{array}$ & $\begin{array}{l}\text { Follicular-small follicle cell } \\
\text { Follicular-small follicle cell } \\
\text { Diffuse-small follicle cell } \\
\text { Diffuse-small lymphocytic } \\
\text { Diffuse-small lymphocytic } \\
\text { Diffuse-small lymphocytic } \\
\text { Diffuse-large undifferentiated cell } \\
\text { Diffuse-large undifferentiated cell } \\
\text { Diffuse-large undifferentiated cell } \\
\text { Diffuse-large undifferentiated cell }\end{array}$ & $\begin{array}{l}\mathbf{M} \\
\mathbf{M} \\
\mathbf{F} \\
\mathbf{F} \\
\mathbf{F} \\
\mathbf{F} \\
\mathbf{M} \\
\mathbf{M} \\
\mathbf{F} \\
\mathbf{M}\end{array}$ & $\begin{array}{l}43 \\
68 \\
59 \\
56 \\
68 \\
63 \\
67 \\
46 \\
67 \\
45\end{array}$ & $\begin{array}{l}\text { Inguinal } \\
\text { Inguinal } \\
\text { Cervical } \\
\text { Tonsil } \\
\text { Cervical } \\
\text { Cervical } \\
\text { Cervical } \\
\text { Axillary } \\
\text { Submandibular } \\
\text { Intra-abdominal }\end{array}$ & $\begin{array}{l}\mathbf{G}, \lambda \\
\mathbf{M}, \mathbf{G}, \lambda \\
\mathbf{M}, \boldsymbol{\lambda} \\
\mathbf{M}, \boldsymbol{\lambda} \\
\mathbf{M}, \boldsymbol{\lambda} \\
\mathbf{M}, \boldsymbol{\kappa} \\
\mathbf{M}, \boldsymbol{\lambda} \\
\mathbf{A}, \mathbf{M}, \kappa \\
\mathbf{G}, \kappa \\
\mathbf{G}, \boldsymbol{\lambda}\end{array}$ & $\begin{array}{l}G, \lambda \\
M, G, \lambda \\
M, \lambda \\
M, \lambda \\
M, \lambda \\
M, \kappa \\
M, \lambda \\
A, \kappa \\
G, \kappa \\
G, \lambda\end{array}$ \\
\hline
\end{tabular}

*Paraffin sections and polyclonal antisera.

†Frozen sections and monoclonal antibodies.

the standard PAP method for cytoplasmic immunoglobulin staining has been reported elsewhere. ${ }^{1}$ In that study it was found that with paraffin sections the IGSS method gave at least equivalent staining to the PAP method when primary (polyclonal) antiserum was used at concentrations up to 200 times less. Apart from any financial or technical advantage, the principal value of such a highly sensitive immunostaining procedure lies in its potential for demonstrating the presence of much smaller quantities of antigen than previously achieved with standard immunoperoxidase techniques.

Our previous experience with immunoperoxidase staining of paraffin sections which was confirmed in this study, indicates that such methods are unable to demonstrate immunoglobulin in the surface membrane of neoplastic lymphocytes. However, from the results described here it is apparent that staining of surface membrane immunoglobulin can be readily achieved in paraffin sections when the IGSS method is employed with polyclonal antisera. Indeed the results obtained were comparable to immunoperoxidase staining of frozen sections with monoclonal antibodies. Moreover, because of the better preservation of tissue architecture in paraffin sections the results were easier to interpret with the IGSS method. The IGSS method also revealed the presence of an additional heavy chain in the surface membrane in one of the cases suggesting that it may be even more sensitive than the immunoperoxidase techniques employing monoclonal antibodies.

The development of immunostaining methods applicable to paraffin sections has several obvious advantages over frozen section techniques. Firstly, it removes the necessity for collecting fresh tissue with the attendant logistical and storage problems. Secondly, preservation of tissue and cellular architecture in paraffin sections enables topographical and morphological analyses that are difficult to accomplish with frozen material. Thirdly, it provides the opportunity to undertake retrospective studies utilising the abundant material already stored in paraffin blocks in most laboratories. Although refinements for improving the sensitivity of the IGSS method are being developed, we believe the much enhanced sensitivity already demonstrated over other immunostaining procedures provides an opportunity to detect a wide range of tissue antigens in paraffin sections in far smaller quantities than hitherto possible. Whilst we have concentrated initially on applications to lymphoma diagnosis preliminary work has already shown its potential for demonstrating antigens in other tumour types.

This study was supported by a grant to CCB from the Yorkshire Regional Cancer Organisation.

\section{References}

' Holgate CS, Jackson P, Cowen PN, Bird CC. Immunogold-silver staining - new method of immunostaining with enhanced sensitivity. J Histochem Cytochem 1983; (in press).

Requests for reprints to: Professor CC Bird, Department of Pathology, University of Leeds, Leeds LS2 9JT, England. 